\title{
Editorial
}

\section{Grand Challenges for Psychiatric Drug Discovery: A Perspective}

\author{
Linda S Brady', Robert B Giffin ${ }^{2}$, Janet Woodcock ${ }^{3}$, Gail H Cassell ${ }^{4}$ and Edward W Holmes ${ }^{5}$ \\ 'Division of Neuroscience and Basic Behavioral Science, National Institute of Mental Health, National Institutes of Health, Bethesda, MD, USA; \\ ${ }^{2}$ Institute of Medicine, National Academy of Sciences, Washington, DC, USA; ${ }^{3}$ US Food and Drug Administration, Rockville, MD, USA; ${ }^{4}$ Eli Lilly \\ and Company, Indianapolis, IN, USA; ${ }^{5}$ A*Star Biomedical Research Council, University of California, La Jolla, CA, USA
}

Neuropsychopharmacology (2008) 33, 2047; doi: 10.1038/npp.2008.54

The Institute of Medicine (IOM) Forum on Drug Discovery, Development, and Translation commissioned a series of papers to tackle the grand challenges facing drug discovery for psychiatric disorders, and to identify novel strategies for the development of new therapeutics. The three papers authored by Conn and Roth (Conn and Roth, 2008; see p. 2048), Carpenter and Koenig (Carpenter and Koenig, 2007; see p. 2061), and Mathew et al (Mathew et al, 2008; see p. 2080) identify opportunities and challenges to translate advances in basic and clinical neuroscience into novel medications to treat mood and anxiety disorders and schizophrenia. Some common themes emerge from these papers.

The authors identify the need to develop novel mechanism of action therapeutics based on validated molecular targets. They observe a high degree of variability in the predictive value of existing preclinical models to assess drug efficacy, and call for more rigorous target validation at each stage along the pathway-from target identification to clinical validation - through clinical trials with appropriate levels of statistical power. The authors summarize the variable degree of predictive value that existing preclinical models have in assessing efficacy in the majority of psychiatric disorders. With the lack of congruence between disease models and symptoms in psychiatric disorders, the development of a strong mechanistic foundation for efficacy and toxicity biomarkers, both preclinical and clinical, is considered crucial for subsequent therapeutic development. The authors identify the pressing need not only for novel prevalidated molecular and cellular targets, but also drug targets that are clinically established.

The authors suggest targeting core phenotypic domains of dysfunction within and across psychiatric disorders, such as cognitive deficits in schizophrenia, sleep disturbances in post-traumatic stress disorder, and suicide across multiple disorders. This approach calls for developing and validating models that translate these clinical phenotypes into experimental endpoints to assess drug efficacy in animals and humans. One example of such an experimental endpoint is fear extinction, which has been successfully modeled in both animals and humans.

The papers cite significant opportunities as well as challenges for system-level approaches to facilitate the translation of basic discoveries into validated drug targets and new models of impaired domains of function. These opportunities have the potential to introduce innovation along the critical path for psychiatric drug discovery.

\section{DISCLAIMER}

The responsibility for the content of these articles rests with the authors and does not necessarily represent the views of the Institute of Medicine or its committees and convening bodies.

\section{REFERENCES}

Carpenter WT, Koenig JI (2008). The Evolution of Drug Development in Schizophrenia: Past Issues and Future Opportunities. Neuropsychopharmacology; doi:10.1038/sj.npp.1301652.

Conn PJ, Roth BL (2008). Opportunities and Challenges of Psychiatric Drug Discovery: Roles for Scientists in Academic, Industry, and Government Settings. Neuropsychopharmacology; doi:10.1038/sj.npp.1301638.

Mathew SJ, Manji HK, Charney DS (2008). Novel Drugs and Therapeutic Targets for Severe Mood Disorders. Neuropsychopharmacology; doi:10.1038/sj.npp.1301639. 